\title{
STANHOPEINAE MESOAMERICANAE (ORCHIDACEAE). III. REESTABLECIMIENTO DE STANHOPEA RUCKERI Y UNA ESPECIE NUEVA: STANHOPEA CONFUSA
}

\author{
GÜNTER GERLACH ${ }^{1} \&$ JORGE BEECHE $^{2}$

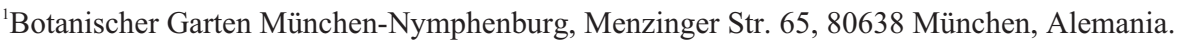 \\ gerlach@extern.lrz-muenchen.de \\ ${ }^{2}$ Jardín Botánico Lankester, Universidad de Costa Rica
}

\begin{abstract}
Stanhopea ruckeri Lindl. is new interpreted and so reestablished. The name S. inodora is transfered to its synonymy, because of investigations in the Lindley Herbarium. Stanhopea gibbosa auct. is found to be not identical with the holotype of $S$. gibbosa Rchb.f., so it was necessary to describe it as a new species. Because of the confusion around its identity, it is named $S$. confusa.
\end{abstract}

RESUMEN. Stanhopea ruckeri se interpreta y se reestablece. Después de examinar el Herbario de Lindley, el nombre S. inodora se transfiere a la sinonimia de S. ruckeri. Se descubrió que Stanhopea gibbosa auct. no es igual que el holotipo de $S$. gibbosa Rchb.f.; por tanto, se describe una especie nueva, que debido a la confusión sobre su identidad se nombra $S$. confusa.

Palabras clave / Key words: Orchidaceae, Stanhopeinae, Stanhopea confusa, Stanhopea gibbosa, Stanhopea inodora, Stanhopea ruckeri, new orchid species, Costa Rica, Panamá, Mesoamérica

En el marco de los trabajos de preparación del tratamiento de Orchidaceae para la Flora Mesoamericana, detectamos varias curiosidades en la taxonomía y la sistemática de las especies de Stanhopeinae. Por ejemplo, algunos problemas en la taxonomía del género Stanhopea, que discutimos a continuación.

\section{Stanhopea ruckeri Lindl. y Stanhopea inodora Lodd. ex Lindl.}

El estatus de $S$. ruckeri Lindl. y de $S$. inodora Lodd. ex Lindl. fue discutido de modos diversos durante los decenios que siguieron a las primeras publicaciones. Lindley (1852), en el primer gran trabajo sobre el género Stanhopea, las trata como especies diferentes pertenecientes a dos grupos distintos. Reichenbach fil. (1858) también las trata como dos especies, pero las pone en un solo grupo junto con $S$. wardii y $S$. graveolens. Pasaron más de cien años hasta que Dodson empezó a estudiar el género cuidadosamente. Para él la cosa se complicó, porque varias especies habían sido descritas sin ser ilustradas. Él fue la primera persona que revisó herbarios en busca de los diferentes tipos. Dodson intentó interpretar los tipos de las especies de
Stanhopea, tomando en cuenta la compleja morfología de las flores. Como pionero, él hizo estudios de plantas vivas y las comparó con las flores herborizadas. Dodson (1963) ilustró S. graveolens como S. ruckeri y la interpretó como posible híbrido entre S. wardii Lodd. ex Lindl. y S. oculata (Lodd.) Lindl., un error perdonable por las dificultades en la taxonomía del género. Unos años después, Kennedy (1975) mencionó que Cal Dodson le informó que $S$. inodora es cercana a, o una forma de, S. ruckeri. Jenny (1989), en su artículo sobre algunas especies de Stanhopea, discute extensamente sobre S. inodora. Él menciona que Reichenbach fil. trabajó mucho con $S$. inodora y publicó un dibujo de ella (Reichenbach 1858). Si se revisa cuidadosamente este dibujo en Xenia Orchidacea, se concluye que no se trata de $S$. inodora, sino de otra especie, muy probablemente $S$. lietzei (Regel) Schltr., la cual tiene el aroma desagradable que Reichenbach mencionó. Jenny (2004) confirma que el dibujo de Reichenbach no representa a $S$. inodora; según él, se trata de $S$. graveolens Lindl. Se sabe que $S$. lietzei (de Brasil) y $S$. graveolens (del sur de México hasta Honduras) tienen indol en su aroma floral, que es responsable del olor desagradable. Las dos se diferencian por el hipoquilo, 


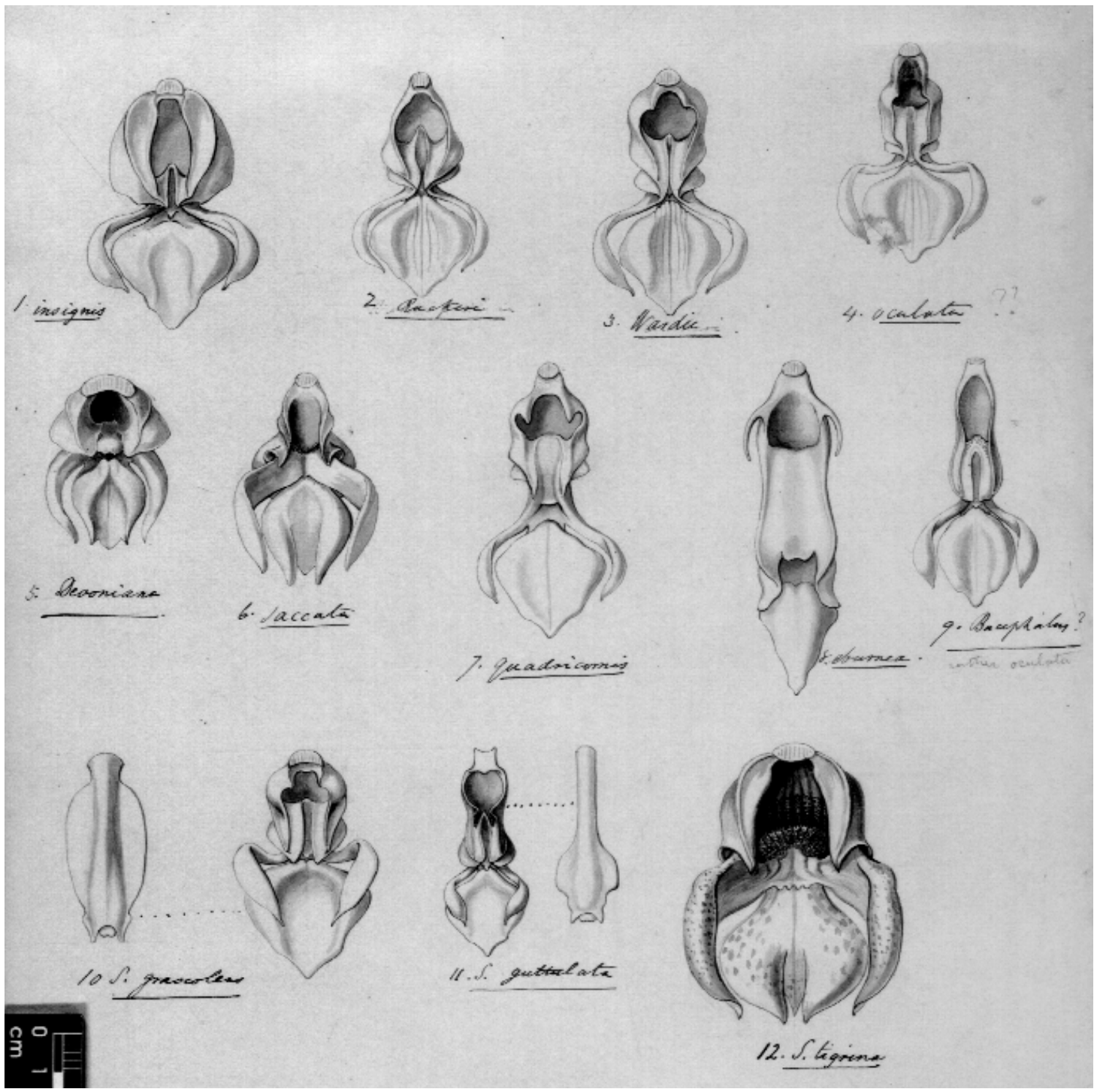

Figura 1. Dibujo de labelos de especies de Stanhopea en el Herbario de Lindley. Reproducido con el gentil permiso del Director y Trustees, the Royal Botanic Gardens, Kew.

el cual en $S$. lietzei es dorsalmente giboso y en $S$. graveolens tiene una parte aplanada. El dibujo de Reichenbach en Xenia presenta una Stanhopea con hipoquilo barrigudo (giboso) en vista lateral; entonces, no se trata de $S$. graveolens, sino de $S$. lietzei.

Al revisar el Herbario de Lindley en Kew, encontramos un dibujo (fig. 1), probablemente de Lindley, montado en forma de exsiccatum, el cual muestra una serie de labelos de especies de Stanhopea (1. S. insignis Frost ex Hook. 1829, 2. S. ruckeri Lindl. 1843, 3. S. wardii Lodd. ex Lindl. 1838, 4. S. oculata (Lodd.)
Lindl. 1832, 5. S. devoniana hort. ex Henshall 1845, 6. S. saccata Batem. 1838, 7. S. quadricornis Lindl. 1838, 8. S. eburnea Lindl. 1832, 9. S. bucephalus Lindl. 1832, 10. S. graveolens Lindl. 1839, 11. S. guttulata Lindl. 1843, 12. S. tigrina Batem. ex Lindl. 1838). Si uno supone que "S. devoniana" es un error ortográfico y que debe tratarse de $S$. devoniensis Lindl., 1838, todas las especies de Stanhopea de esta lámina fueron descritas antes del inicio del año 1844. En este tiempo, S. inodora no se conocía; Lindley la publicó más de un año después. El labelo de $S$. ruck- 


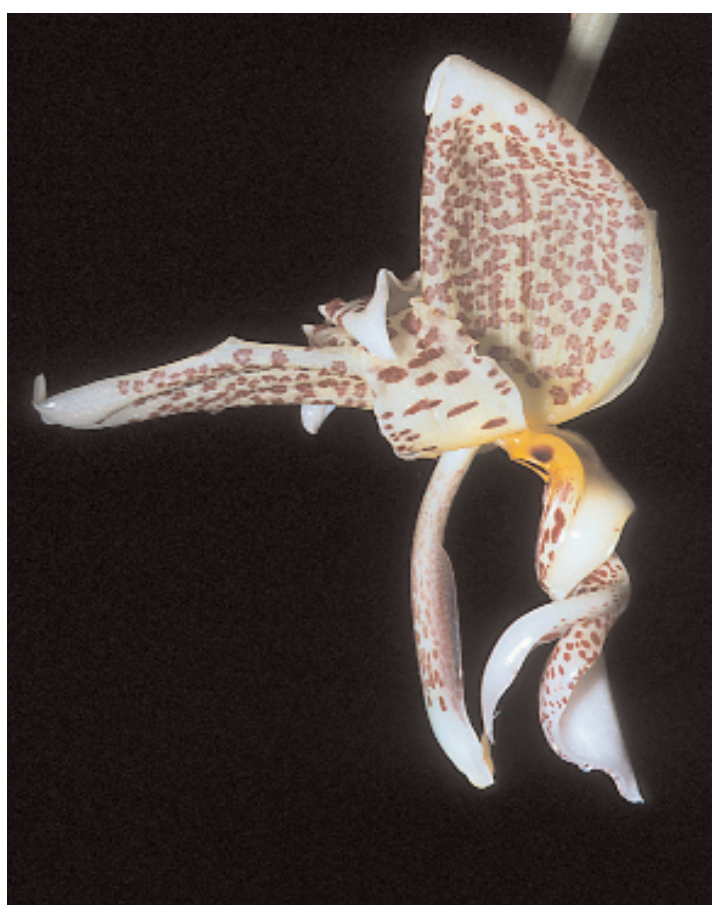

Figura 2. Stanhopea ruckeri, 99/3320. México: Edo. Veracruz. Forma bien maculada. Foto G. Gerlach.

eri muestra allí un diente central muy pronunciado, que sobresale en gran parte desde la cavidad del hipoquilo. Este diente es muy característico y no es tan grande en las otras especies ilustradas en esta lámina. Stanhopea ruckeri fue descrita por Lindley en 1843; él menciona este diente pronunciado: “... and by the presence of a very strong inflexed tooth, in which the wide, not closed up, fissure of the apex of the hypochilium terminates". Otra información importante es el color pálido. En 1845, Lindley describió $S$. inodora con material de Loddiges, quien importó cierto número de plantas de México. La publicación incluye también un dibujo muy informativo que muestra igualmente este diente del hipoquilo. Aquí es interesante que Lindley señala que las flores también son pálidas. En la descripción de S. inodora, el autor la compara con $S$. graveolens y la distingue de ésta, pero no menciona nada sobre $S$. ruckeri. ¿Pudo ser que él olvidó la existencia de su S. ruckeri? ¿O que él no tomó en cuenta que su $S$. ruckeri es tan cercana a S. inodora? De todos modos, para nosotros es claro que se trata de una sola especie y, según el Código Internacional de Nomenclatura Botánica, el nombre

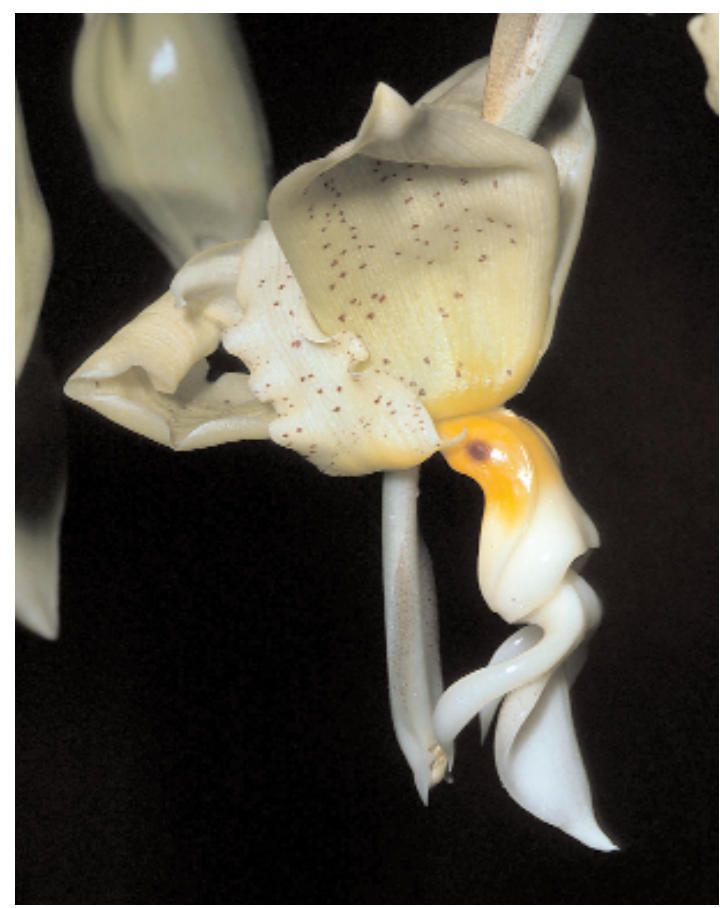

Figura 3. Stanhopea ruckeri, 87/3842. Sin origen conocido. Forma típica, casi sin puntos y manchas. Foto G. Gerlach.

correcto debe ser S. ruckeri y S. inodora es un sinónimo.

Stanhopea ruckeri ahora se presenta como una especie muy variable en el color, aunque la mayoría de los clones muestran flores de un amarillo muy pálido (fig. 2, 3). Raramente existen clones muy llamativos con los sépalos y los pétalos densamente manchados de rojo marrón. Características importantes de la especie son el labelo poco geniculado y un diente pronunciado que sobresale en gran parte desde la cavidad del hipoquilo. La inflorescencia es laxa y tiene normalmente cuatro flores.

Jenny (2004) menciona el tipo presente en el Natural History Museum (BM) en Londres, pero no se encontró el espécimen. Hay un ejemplar del Herbario de Reichenbach que tiene dos especies de Stanhopea: S. ruckeri y S. oculata, como menciona Jenny en su monografía. Quizás él se equivocó y citó este ejemplar como material de Lindley. Sin duda, este espécimen muestra la verdadera S. ruckeri al lado de S. oculata. En un artículo reciente (Kramer 2000) se discute el misterio de $S$. ruckeri. Para nosotros la especie se presenta muy clara, con las características 
descritas arriba. Por faltar material original de Lindley, aquí se designa un lectotipo.

Stanhopea ruckeri Lindl., Edward's Bot. Reg. 29: sub. t.44. 1843.

Lectotipo (designado aquí): Dibujo del labelo por John Lindley (K-L).

FIG. 1.

DistRIBUCión. La especie se encuentra desde México (Veracruz) hasta el norte de Nicaragua (Jinotega).

Especímenes examinados: MéXico. Chiapas: Ocosingo, Crucero Corozal, $144 \mathrm{~km}$ al SE de Palenque, por la carretera a Marquéz de Comillas, 150 m, 21.7.1989, Soto, Purrata, Martínez 5716 (AMO). Oaxaca: Camino Oaxaca Pto. Escondido, Hágsater 1195 (AMO). Veracruz: near Zacuapán, $19^{\circ} 12^{\prime} \mathrm{N}, 96^{\circ} 52 \mathrm{~W}$, ca. $900 \mathrm{~m}, 7.6 .1935$, Nagel 3327 (US); "Barranca Seca" near Tezonapa, $18^{\circ} 36^{\prime} \mathrm{N}, 96^{\circ} 41^{\prime} \mathrm{W}, 400$ m, 29.8.1936, Nagel (US); Amatlán de los Reyes, sobre el Río Negro en el Ejido Guadalupe, 23.7.1993, Huerta Alvízar 91 (AMO); a $200 \mathrm{~m}$ del puente San Isidro en Maloxtla, 5.7.1993, Huerta Alvizar 85 (AMO); Amatlán de los Reyes, unión de los ríos Chinicuilapa y Chilpanapa, cerca del Río Negro en el Rancho Chilpanapa, 5.7.1993, Huerta Alvizar 86 (AMO); Amatlán de los Reyes, sobre el Río Maloxtla, a $50 \mathrm{~m}$ del puente San Isidro, 20.7.92, Huerta Alvizar 78 (AMO); Amatlán de los Reyes, a la orilla del río, a $200 \mathrm{~m}$ del puente San Isidro en Maloxtla, 23.7.1993, Huerta Alvizar 90 (AMO); near Jalapa, floreció en cult. 8/1901, Rose \& Hay 308 (US); near Jalapa, floreció en cult. 18.8.1901, Rose \& Hay 6194 (US). Guatemala. Alta Verapaz: Unión Río Chicsoy con Río Quixjal, ca. 900 m, 10.5.1987, Día s.n. (UVAL). HoNDURAS. Morazán: Zamorano, 800 m, 5.1944, Rodríguez 2129 (F); Morazán: Zamorano, 800 m, 25.6.1945, Rodríguez 3115 (F); sine loco, 28.8.1974, Rodríguez 1450 (USJ). NICARAGUA. Jinotega: Bocaycito, cerca a Mt. Peñas Blancas, 600-1000 m, cult. bajo Whitten $1055+1057+1058+94066$ (FLAS); Macizos de Peñas Blancas, $1400 \mathrm{~m}$, Heller $7251+7233+7155$ (SEL); Pantasmi River, Heller 1838 (solamente dibujo y descripción) (SEL).

\section{Stanhopea gibbosa Rchb.f.}

En 1869 Reichenbach filius describió una Stanhopea con el nombre S. gibbosa. La descripción es corta; se refiere a una planta obtenida de los invernaderos de Veitch \& Sons en Inglaterra. Reichenbach nunca publicó un dibujo de la especie; así, la interpretación de la misma ha sido muy vaga, especialmente para los investigadores que sufrieron por la clausura del Herbario de Reichenbach durante 25 años después de su muerte, tal como él lo ordenó en su testamento. Como se mencionó antes, fue Dodson quien trabajó primero con la taxonomía del género Stanhopea. Él tipificó muchas de las especies presentes en el Herbario del Naturhistorisches Museum Wien (W-R), en Viena, Austria. Allí se encuentra el tipo de $S$. gibbosa.

Hay tres especímenes con el nombre $S$. gibbosa en el Herbario de Reichenbach; uno con una flor prensada y un dibujo al lado obtenido por Veitch \& Sons (\# 24450, fig. 4, 5), otro con una inflorescencia de dos flores, sin más información (\# 33864), y un tercero que consiste solamente de acuarelas de dos especies ( $S$. gibbosa y $S$. costaricensis). Actualmente, estos dibujos montados están separados: 24481a es $S$. gibbosa, 24481b es $S$. costaricensis. En 1963 Dodson anotó que probablemente $S$. gibbosa era un híbrido natural entre $S$. costaricensis y $S$. wardii y seleccionó como tipo el ejemplar 24450. Después, él preparó dibujos que ahora están en la colección de Marie Selby Botanical Gardens (SEL), sin tomar en cuenta que no representan la misma especie que muestra el tipo, sino otra especie que sin duda es la misma de la acuarela 24481a. Hasta entonces todos los investigadores de Stanhopea interpretaron S. gibbosa con base en estos dibujos.

Si uno lee el protólogo con mucho cuidado, encuentra bajo la descripción del hipoquilo la frase "pectore argute praecincto", que significa que el hipoquilo exhibe ventralmente una cintura aguda. El dibujo del tipo muestra muy bien esta característica, que no está presente en los otros dibujos de Reichenbach ni en los dibujos de Dodson. En otras palabras, el mismo Reichenbach mezcló dos especies diferentes y les asignó el nombre $S$. gibbosa; posteriormente, los investigadores siguieron el camino más fácil: Usar los mejores dibujos autorizados por el autor de la especie.

Al parecer, Reichenbach vió por lo menos dos clones de la especie que no era $S$. gibbosa, todos recibidos de Veitch: el primero en agosto de 1878 (Aug. 78), con la anotación adicional "C.R. Endres"; el segundo en junio de 1880 (Juni 80). En el de agosto de 1878, obviamente recolectado por Endrés en Costa Rica, Reichenbach anotó: "Lippe weisslich, am Grunde seitlich mit rothem Aug, Sep. \& Tep ...” 


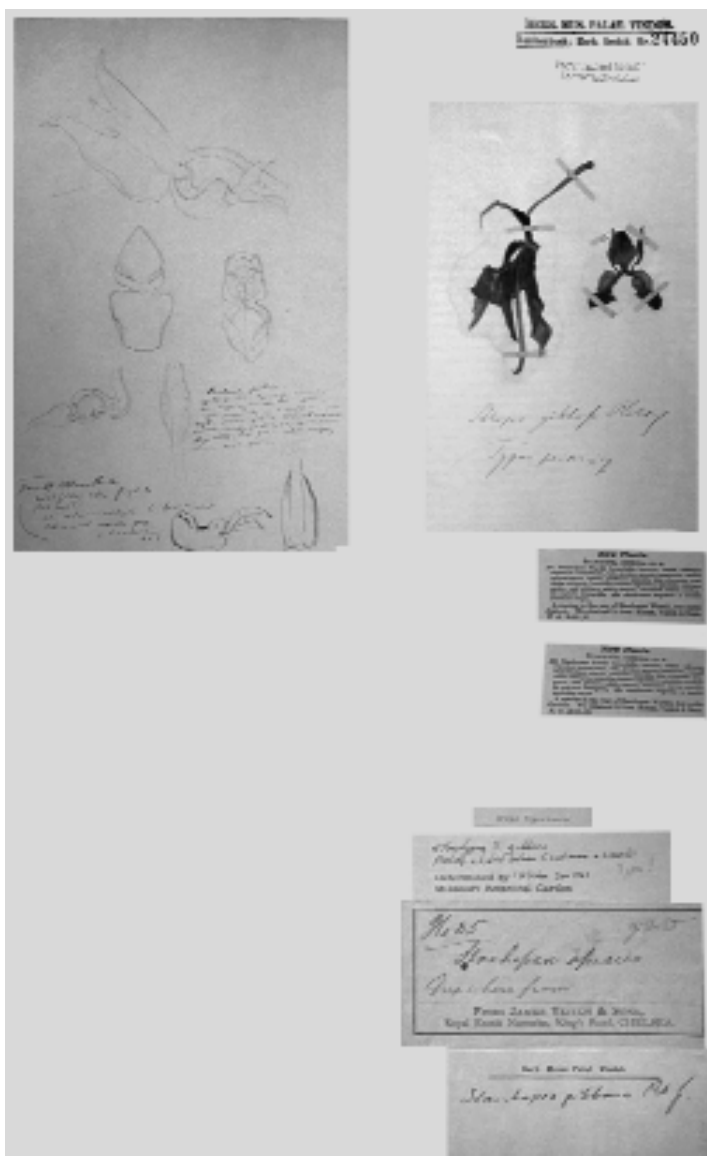

Fig. 4. Tipo de Stanhopea gibbosa. Herbario de Reichenbach \# 24450 (W). Reproducido con la autorización del Curador, Herbario del Museo de Historia

(labelo blanquecino, en la base con ojo rojo, sépalo y tépalo ...). Que esta especie fue recolectada en Costa Rica se confirma por el hallazgo posterior de plantas en la vertiente caribe de este país y en la adyacente de Panamá. En muchos casos tiene flores blanquecinas o de color crema con ojos rojo oscuro, pero también hay clones manchados de rojo.

Regresemos a la interpretación del tipo. Resulta que el origen de S. gibbosa no se conoce! Se trata de una Stanhopea con el hipoquilo giboso y con una cintura ventral bien aguda. Dorsalmente el hipoquilo no tiene ningún adelgazamiento o talle. Eso significaría que los dibujos en la muestra \# 24481a no tienen nada que ver con el tipo de $S$. gibbosa, porque su hipoquilo no está fuertemente arqueado formando una giba (¡de

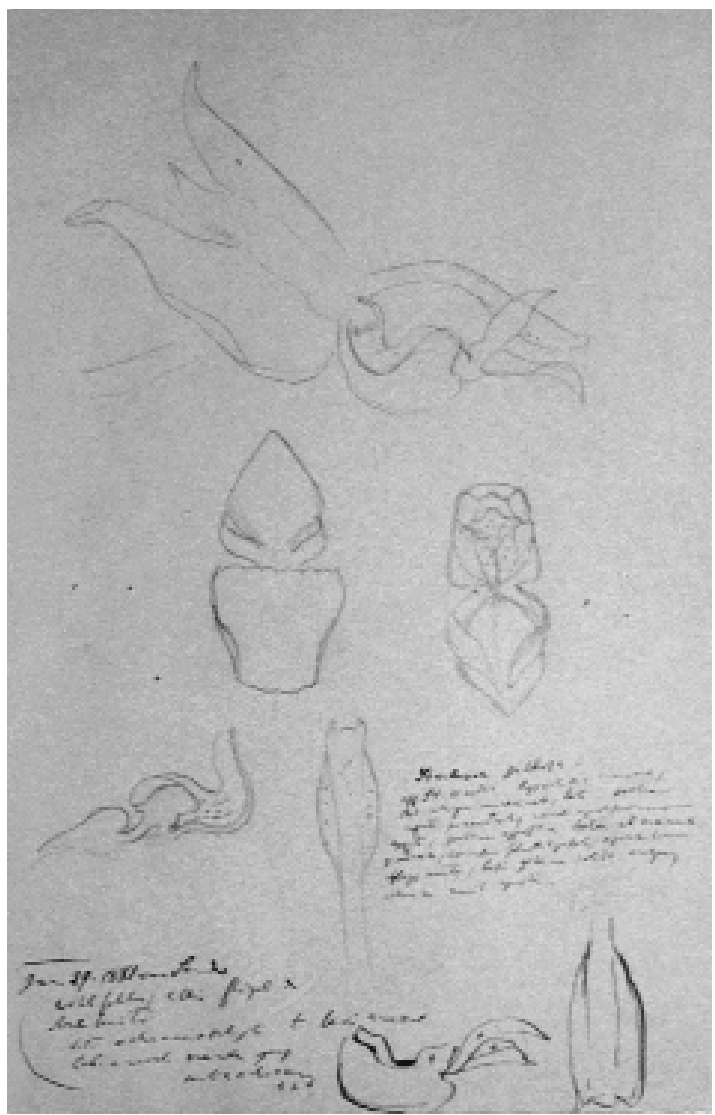

Fig. 5. Tipo de Stanhopea gibbosa. Detalle del dibujo. Herbario de Reichenbach \# 24450 (W). Reproducido con la auto-rización del Curador, Herbario del Museo de Historia Natural, Viena.

aquí el nombre!), sino que es recto, con una depresión muy leve en el vientre. Al comparar la flor dibujada en el espécimen tipo, llegamos a la conclusión de que se trata de la misma especie que Rolfe describió después, en 1898, como $S$. impressa. En consecuencia, es necesario nombrar como $S$. gibbosa a una especie ecuatoriana y colombiana y poner el nombre $S$. impressa en la sinonimia de $S$. gibbosa. Por tanto, la especie de Costa Rica y Panamá dibujada en la muestra \# 24481a no tiene nombre y a continuación se describe como nueva para la ciencia.

Stanhopea confusa G.Gerlach \& Beeche, spec. nova (=S. gibbosa auct., non Rchb.f.)

TIPO: CosTA RICA: Cartago; Turrialba, vertiente este 


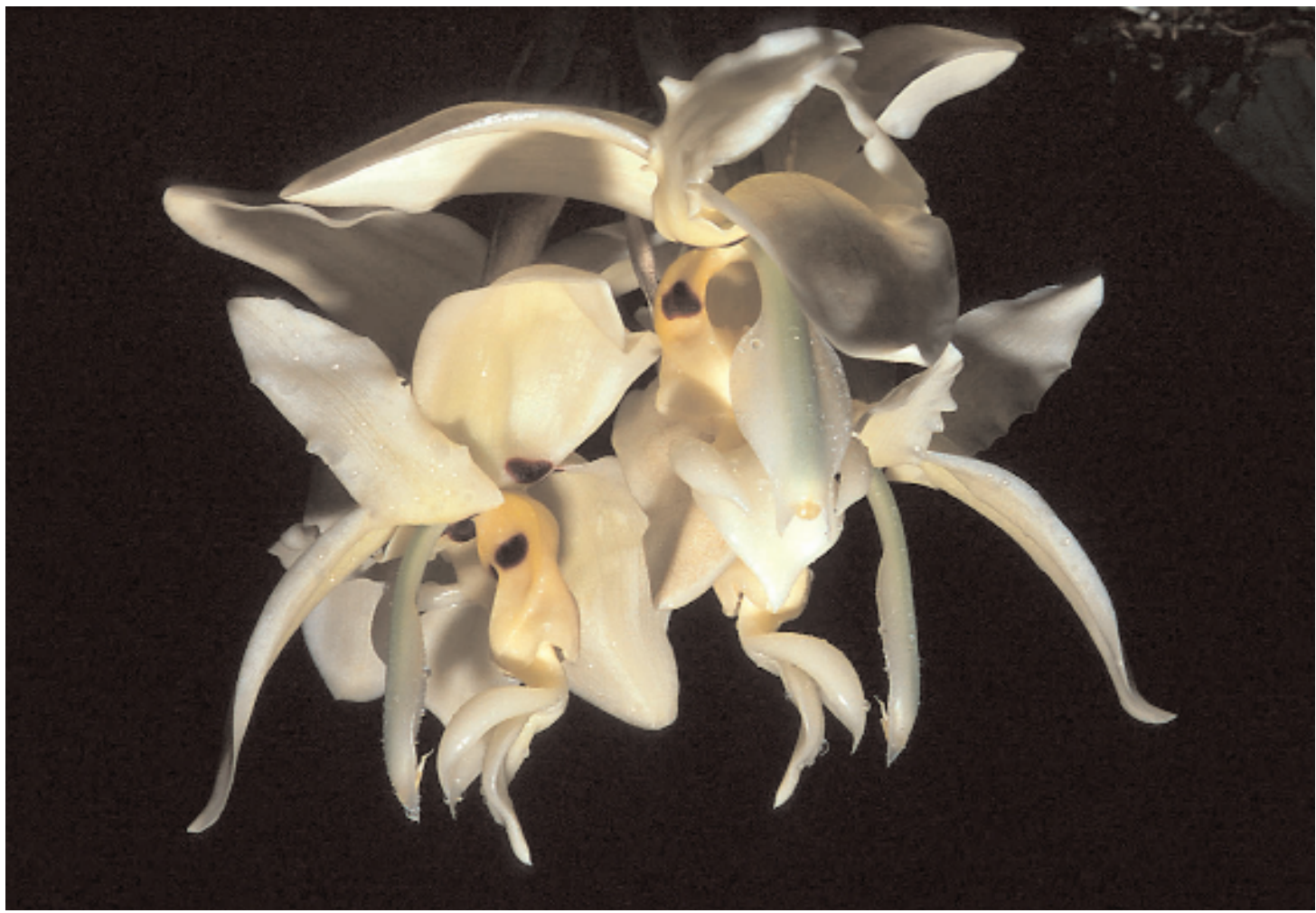

Figura 6. Stanhopea confusa, 93/3084, TIPO. Costa Rica. Cartago: Cordillera de Talamanca. Foto G. Gerlach.

de la Cordillera de Talamanca, entre Grano de Oro y Moravia de Chirripó, selva siempreverde caribe en un valle intermontano, ca. 800 - $900 \mathrm{~m}, 13.7 .1993$, leg. C.K. Horich s.n., floreció en el invernadero cálido del Jardín Botánico de Múnich-Nymphenburg, Alemania, sept 2000, bajo el número 93/3084 (Holotipo: USJxxxxx).

FIG. 6-9.

Differt ab speciebus omnibus subgeneris Stanhopea costaricensibus et panamensibus ( $S$. costaricensis, S. maduroi, S. panamensis, S. wardii, S. warscewicziana) combinatione characteribus: hypochilum subrectum (non geniculatum); aspectu laterale subrectangulare (in basi paullo angustior, in basale tertia parte paullo praecinctum); aspectu dorsale item subrectangulare (ad apicem subamplificatum). Insuper differt a proxime affini Stanhopea costaricensi floribus minoribus (plus minusve longitudo labelli $5,4 \mathrm{~cm}$ versus $6,0 \mathrm{~cm}$ ) et hypochilo paullo praecincto.

Pseudobulbos ovoides hasta globosos, poco surcados, totalmente cubiertos por varias vainas papiráceas, éstas hasta $17 \mathrm{~cm}$ de largo; hoja peciolada, pecíolo hasta $13 \mathrm{~cm}$; lámina $41 \mathrm{~cm}$ de largo y $13,5 \mathrm{~cm}$ de ancho, coriácea, verde oscura; inflorescencia gruesa, cubierta por varias vainas papiráceas punteadas de negro, $13 \mathrm{~cm}$ de largo, con hasta 6 flores; pétalos y sépalos de color crema en la base, con una (sépalos) o dos (pétalos) manchas (ojos) púrpuras; sépalo dorsal elíptico, 7,0 cm de largo y $3,7 \mathrm{~cm}$ de ancho, sépalos laterales 7,1 x 4,6 cm, connados $1,4 \mathrm{~cm}$ en la base; pétalos oblongo-lanceolados, con márgenes ondeados, $6,1 \times 2,2 \mathrm{~cm}$; labelo de color amarillo pálido, con dos manchas grandes (ojos) vino tinto en el hipoquilo, el resto de color cremoso, $5,6 \mathrm{~cm}$ de largo y $3,2 \mathrm{~cm}$ de ancho en la distancia de los cuernos del mesoquilo; hipoquilo 2,8 cm de largo, 2,3 cm de ancho y $1,6 \mathrm{~cm}$ de grueso, con una apertura oval $1,3 \mathrm{~cm}$ de ancho y 1,0 $\mathrm{cm}$ de largo, muy poco geniculado, con una leve cintura en el tercio basal, en vista lateral casi rectangular (en la base un poco menos ancho que cerca del ápice), en vista dorsal igualmente rectangular, hacia el ápice poco alargado; mesoquilo con dos cuernos arqueados, semirredondos, $2,8 \mathrm{~cm}$ de largo y $0,7 \mathrm{~cm}$ de diámetro, 


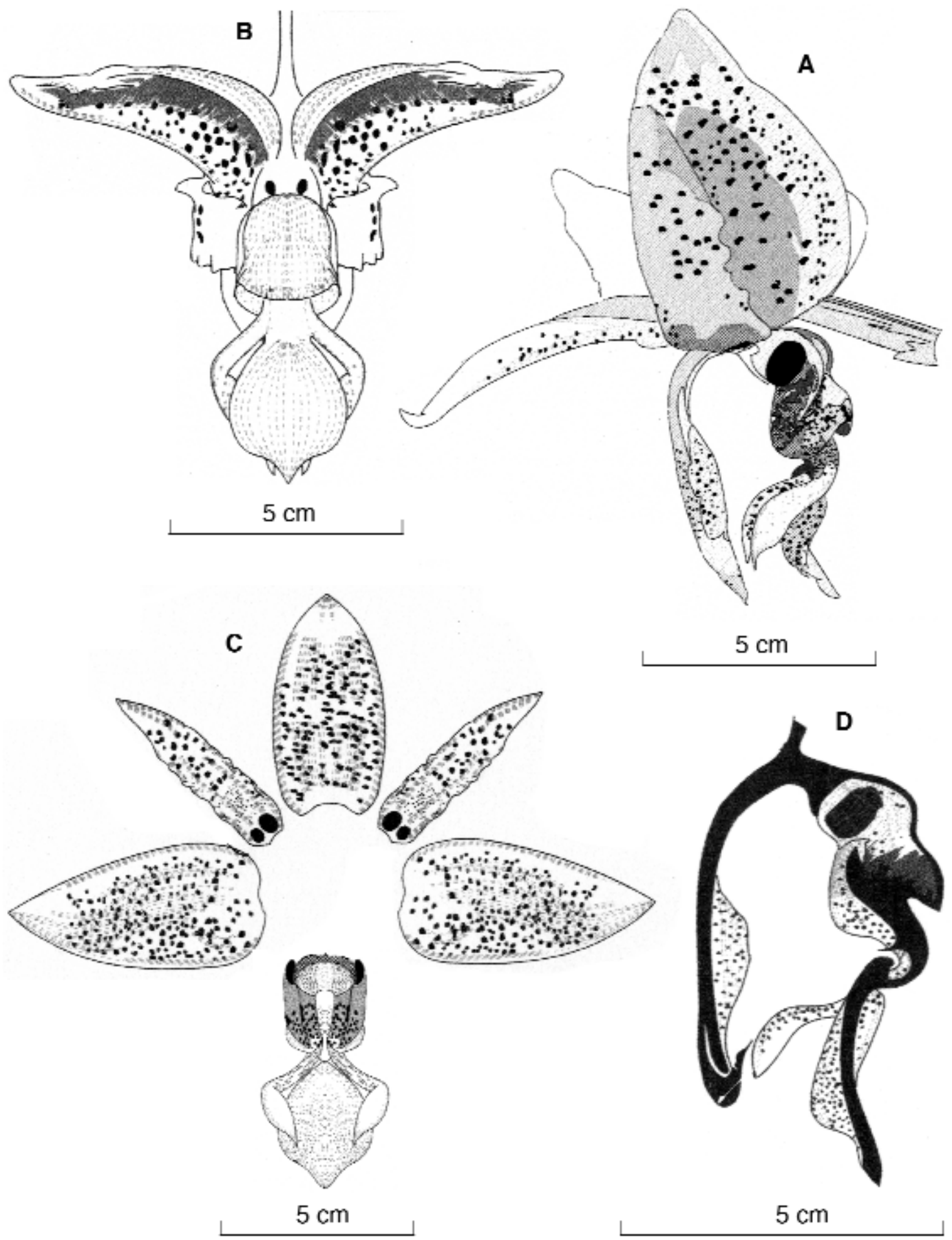

Figura 7. Stanhopea confusa G. Gerlach \& Beeche. A - Flor, vista lateral. B - Flor, vista abaxial. C - Perianto seccionado D - Columna y labelo, sección longitudinal. Dibujo de J. Beeche. 


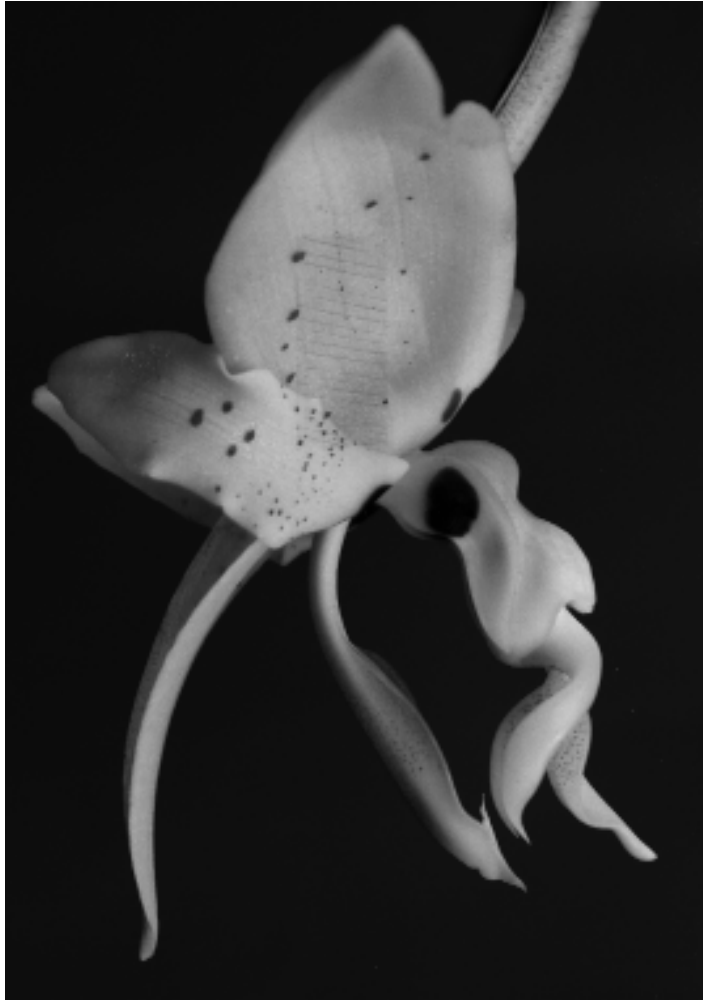

Figura 8. Stanhopea confusa G. Gerlach \& Beeche, JB 310. Vista lateral de la flor (testigo no preservado).

ápices apiculados, distancia entre las puntas $1,4 \mathrm{~cm}$; epiquilo casi redondo, con apículo poco pronunciado, $2,3 \times 2,4 \mathrm{~cm}$; columna de 5,2 cm de largo, levemente aplanada, en la base débilmente arqueada, hacia el ápice con alas laterales, con dos dientes apicales, con las alas llegando hacia 2,0 cm de largo.

DiAgNóstico DIfERENCIAL. Se diferencia de todas las demás especies del subgénero Stanhopea de Costa Rica y Panamá (S. costaricensis, S. maduroi, S. panamensis, S. wardii, S. warscewicziana) por la combinación de caracteres del hipoquilo: casi recto, muy poco geniculado, rectangular en vista lateral, con una leve cintura en el tercio basal, en vista dorsal igualmente rectangular, hacia el ápice poco alargado. De su próxima vecina, $S$. costaricensis, se diferencia por las flores más pequeñas (largo del labelo $5,4 \mathrm{~cm}$ versus $6,0 \mathrm{~cm}$ ) y el hipoquilo con menor cintura.

Distribución. La especie se extiende desde Nicaragua, pasando por el este de Costa Rica (especialmente la provincia de Cartago), hasta el nordeste

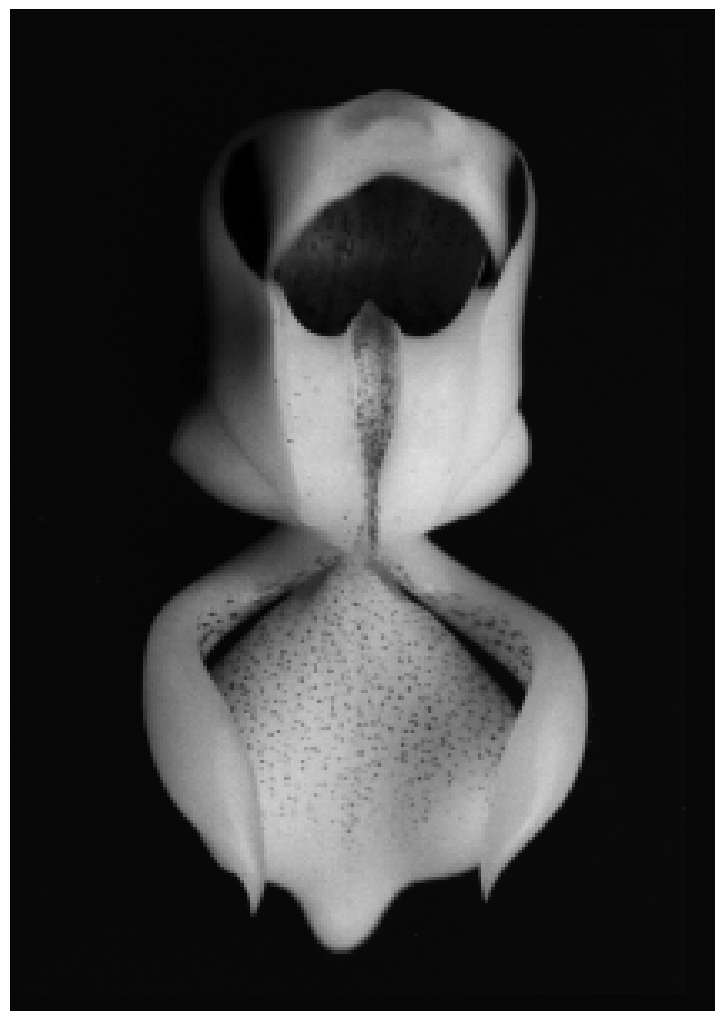

Figura 9. Stanhopea confusa G. Gerlach \& Beeche, JB 310. Vista adaxial del labelo (testigo no preservado).

de Panamá (Prov. Bocas del Toro). Las pocas muestras con origen conocido indican que crece a alturas entre 600 y $1220 \mathrm{~m}$. Seguramente habrá que ampliar este ámbito, porque hasta ahora los datos son muy incompletos.

ESPECÍMENES EXAMINADOS: COSTA RICA. Heredia: Cariblanco; camino a Colonia Virgen del Socorro, $850 \mathrm{~m}$, jul 1995, Blanco 51 (USJ). Cartago: región de Río Pacuare, 600-800 m, Horich, cult. bajo 92/3249 (M); cerca a La Suiza de Turrialba, ca. $700 \mathrm{~m}$, Horich, cult bajo 93/3078 (M); San Rafael de Oreamuno, 1.9.1945, Echeverría 30 (F). PANAMÁ. Chiriquí: 4000 feet [1220 m], Powell 103 (BM); Fortuna, Dressler, cult. bajo Whitten 94082 (FLAS); Fortuna, Williams, cult. bajo Whitten 1053 (FLAS). Panamá: Cerro Campana, ca. 900 m, Atwood, cult. bajo Whitten 1051 (FLAS).

ETIMOLOGÍA. El epíteto latino confusa se refiere a la confusión que existió durante mucho tiempo en torno a la identidad de esta especie.

La especie es notablemente variable en su coloración (González \& Mora-Retana 1996). El color de 
fondo normalmente es crema o amarillo pálido. Muchos de los clones observados son unicolores en el fondo, con manchas redondas (ojos) de color rojo (vino tinto) hasta negro en las bases de sépalos, pétalos y labelo. También existen clones con puntos o manchas en las partes florales; aun cuando las manchas son muy densas, la parte alrededor del "ojo" tiene el color de fondo. En todos los clones investigados existe un "ojo" oscuro en la base del labelo.

En gran parte de la literatura y en los herbarios, $S$. confusa aparece mezclada con $S$. costaricensis. Normalmente, $S$. costaricensis es más grande que $S$. confusa. Los datos publicados sobre el tamaño de $S$. confusa (Mora-Retana \& González 1996, citada como S. gibbosa) coinciden muy bien con nuestras investigaciones. Sin embargo, no parece creíble la longitud del labelo de $S$. costaricensis que ellas comunican: entre $4,5 \mathrm{~cm}$ y $7,0 \mathrm{~cm}$. Es probable que una $S$. confusa se ocultara entre los ejemplares de $S$. costaricensis, modificando los datos. Otra diferencia es la presencia del "ojo" oscuro en la base del hipoquilo de todos los clones de S. confusa investigados. En S. costaricensis hay muchos clones que carecen del "ojo". En S. costaricensis el hipoquilo muestra una cintura pronunciada; esta cintura existe, pero menos evidente, en $S$. confusa.

El aroma floral de $S$. confusa nos recuerda el del chocolate. Investigaciones del aroma floral no muestran una diferencia entre $S$. costaricensis y $S$. confusa, tal como fue publicado por Whitten \& Williams (1992). La composición química está dominada por eucaliptol $(73,0-84,4 \%)$, seguido por b-mirceno $(7,4-$ $14,5 \%)$, limoneno $(0,1-7,1 \%)$, a-pineno $(1,2-5,0 \%)$, b-pineno $(0,3-1,2 \%)$ y (E)-ocimeno (0-1,5\%). Según Dodson (1965), la especie es polinizada por Eulaema meriana; el mismo autor nombra a Eulaema seabrae como polinizador de S. costaricensis. Es posible que las dos especies no hayan sido correctamente identificadas, por problemas en taxonomía. Nuevas observaciones de campo sobre la polinización de las dos especies se requieren urgentemente, para constatar las diferencias y el probable aislamiento génetico de ambos taxa debido a diferentes polinizadores.

Agradecimientos. A Franz Schuhwerk (Botanische Staatssammlung München, Alemania) por traducir la descripción diferencial al latín, a Carlos O. Morales (Universidad de Costa Rica) por comentarios muy valiosos sobre el manuscrito y por mejorar la comprensión del texto, a Corina Gerlach por su ayuda en las correcciones del texto, a los curadores Ernst Vitek y Bruno Wallnöfer (Naturhistorisches Museum, Viena, Austria) por su apoyo con el Herbario de Reichenbach, a Phillip Cribb y Jeffrey Wood (Royal Botanic Gardens, Kew, Inglaterra) por la ayuda con el Herbario de Lindley y a Roman Kaiser (Givaudan Schweiz AG, Dübendorf, Suiza) por los análisis de los aromas florales.

\section{LITERATURA CITADA}

Dodson, C.H. 1963. The Mexican Stanhopeas. Amer. Orch. Soc. Bull. 32: 115-129.

Dodson, C.H. 1965. Agentes de polinización y su influencia sobre la evolución en la familia Orquidácea. Univ. Nac. Amazonia Peruana, Inst. General de Investigaciones. $128 \mathrm{p}$.

González L., M.I. \& Mora-Retana, D.E. 1996. El color de las flores en la taxonomía de cuatro especies de Stanhopea (Orchidaceae). Rev. Biol. Trop. 44(2A): 525539.

Jenny, R. 2004. The genus Stanhopea. 1th part. - S. anfracta to S. napoensis. Caesiana 21: 1-145.

Jenny, R. 2004. The genus Stanhopea. 2th part. - S. nigripes to $S$. xytriophora. Caesiana 22: 146-291.

Jenny, R. 1989. Die Gongorinae, 7. Stanhopea, Teil VI: Stanhopea embreei, Stanhopea impressa, Stanhopea frymeri, Stanhopea inodora und Stanhopea lietzei. Orchidee (Hamburg) 40(5): 158-163.

Horich, C.K. 1974. The Costa Rican Stanhopea spcs. Orch. Dig. 38 (2): 108-113.

Kennedy, G.C. 1975. The Stanhopeas of Mexico. Orch. Dig. 39 (5): 178-182.

Kramer, R. 2000. The mystery surrounding Stanhopea ruckeri Lindl. Orch. Australia 12(4): 52-53.

Lindley, J. 1843. Stanhopea ruckeri. Bot. Reg. sub t. 44.

Lindley, J. 1845. Stanhopea inodora. Bot. Reg. t. 65.

Lindley, J. 1852. Stanhopea. Fol. Orchidacea 1-8.

Mora-Retana, D.E. \& González L., M.I. 1996. Variabilidad floral de cuatro especies de Stanhopea (Orchidaceae). Rev. Biol. Trop. 44(2A): 517-523. 